Journal of Intelligent Material Systems \& Structures

\title{
Analytical modelling of the shape memory effect in SMA beams with rectangular cross section under reversed pure bending
}

\begin{tabular}{|r|l|}
\hline Journal: & Journal of Intelligent Material Systems and Structures \\
\hline Manuscript ID & JIM-20-366.R1 \\
\hline Manuscript Types: & Original Article \\
\hline Author: & n/a \\
\hline Komplete List of Authors: & \begin{tabular}{rl|} 
Radi, Enrico; Università degli Studi di Modena e Reggio Emilia Facoltà di \\
Ingegneria Sede di Reggio Emilia, DISMI
\end{tabular} \\
\hline & $\begin{array}{l}\text { Shape Memory, Analytical modeling, Pure bending, Cyclic loading, } \\
\text { Rectangular cross-section, Martensite reorientation }\end{array}$ \\
\hline & $\begin{array}{l}\text { An analytical model is developed for a prismatic SMA beam with } \\
\text { rectangular cross section subjected to alternating bending at } \\
\text { temperature below the austenitic transformations. The loading path } \\
\text { consists in a loading-unloading cycle under bending and reversed } \\
\text { bending. Two opposite martensitic variants take place, whose volume } \\
\text { fractions evolve linearly with the axial stress. Different Young's moduli } \\
\text { are taken for the austenitic and martensitic phases. As the bending } \\
\text { moment is increased, the martensitic transformation starts from the top } \\
\text { and bottom and then it extends inwards. If the maximum applied } \\
\text { bending moment is large enough, then the complete Martensitic } \\
\text { transformation takes place at the upper and lower parts of the cross } \\
\text { section. During unloading and reversed bending, reorientation of the } \\
\text { Martensite variants into the opposite ones takes place starting from the } \\
\text { boundary between the fully martensitic region and the intermediate } \\
\text { transforming region. Special attention is devoted to calculate analytically } \\
\text { the axial stress and Martensite variant distributions within the cross } \\
\text { section at each stage of the process. A closed form moment-curvature } \\
\text { relation is provided for loading and elastic unloading and in integral form } \\
\text { for the rest of the process. The approach is then validated by comparison } \\
\text { with analytical results available in the literature. }\end{array}$ \\
\hline Abstract \\
\hline
\end{tabular}

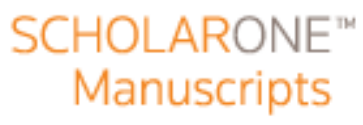




\title{
Analytical modelling of the shape memory effect in SMA beams with rectangular cross section under reversed pure bending
}

\author{
Enrico Radi \\ Dipartimento di Scienze e Metodi dell'Ingegneria, Università di Modena e Reggio Emilia, \\ Via Amendola 2, I-42122 Reggio Emilia, Italy.Email: enrico.radi@unimore.it
}

\begin{abstract}
An analytical model is developed for a prismatic SMA beam with rectangular cross section subjected to alternating bending at temperature below the austenitic transformations. The loading path consists in a loading-unloading cycle under bending and then under reversed bending. Two opposite martensitic variants take place, whose volume fractions evolve linearly with the axial stress. Different Young's moduli are taken for the austenitic and martensitic phases. As the bending moment is increased, the martensitic transformation starts from the top and bottom and then it extends inwards. If the maximum applied bending moment is large enough, then the complete Martensitic transformation takes place at the upper and lower parts of the cross section. During unloading and the following reversed bending, reorientation of the Martensite variant into the opposite one takes place starting from the boundary between the fully martensitic region and the intermediate transforming region. Special attention is devoted to calculate analytically the axial stress and Martensite variant distributions within the cross section at each stage of the process. A closed form moment-curvature relation is provided for loading and elastic unloading and in integral form for the rest of the process. The approach is then validated by comparison with analytical results available in the literature.
\end{abstract}

Keywords: Analytical modeling, Pure bending, Rectangular cross-section, Shape-memory alloy, Phase transformation, Martensite reorientation, Cyclic loading.

\section{Introduction}

Due to their peculiar characteristics, such as the shape memory effect and the superelastic behavior, SMA beams, rods and wires are effectively employed in smart sensors and actuators, as well as in energy dissipating devices for the control of structural vibrations due to wind or seismic excitations. Indeed, a large hysteresis loop similar to that exhibited by ductile metals is observed in a martensitic SMA bar cyclically loaded at low temperatures, which is due to the reorientation process between 
martensitic variants rather than to phase transformation or dislocation glide mechanisms. As a consequence, SMAs are able to display a much higher fatigue resistance than plastically deformed metals and thus they can efficiently replace elastic-plastic hysteretic devices in passive control and seismic retrofit of building and bridges (Dolce et al., 2000; Dolce and Cardone, 2001; DesRoche and Smith, 2004; Song et al., 2006; Ozbulut et al., 2011; Fang and Wang, 2020). A practical example of application of the SMA energy dissipation properties is given by the innovative yielding dissipater device recently proposed by Zibasokhan et al. (2019) for seismic protection of concentrically braced structures, which is able to transform the axial force into pure bending in the dissipating plates. The device is fabricated by a set of ductile steel plates under pure bending, and it can be efficiently improved by exploiting the shape memory effect of SMA.

Usually, dissipating SMA device are cyclically loaded alternately under tension and compression. However, the occurring of buckling in slender SMA bars and wires under compression restricts their applications and requires additional devices to prevent it. Therefore, the special properties of SMA bars are better exploited under bending rather than under tension and compression. In order to overcome the buckling problem, Choi et al. 2009 proposed the use of a superelastic SMA bars in bending as seismic dampers and restrainers for bridges and proved their ability in reducing the openings at the internal hinges and the pounding force on abutments. Later, Choi et al. (2019) investigated the self-centering and damping capacity of SMA bars in bending. They showed that a martensitic SMA bar exploiting the shape memory effect displays a higher energy dissipation and thus a better seismic efficiency than a superelastic SMA bar, while the latter provides better displacement recovery and self-centering capability. Although a residual strain usually remains in a martensitic SMA bar after unloading, it can be easily removed upon heating.

The dissipation capabilities of SMA seismic devices under cyclic loading of alternating sign remarkably increase if the shape memory effect is exploited, rather than the superelastic behavior. Indeed, a larger amount of energy is dissipated during each cyclic reorientation process between positive and negative martensitic variants if no intermediate austenitic transformation is triggered (Wilson and Wesolowsky, 2005). In these applications, a clear under-standing of the fatigue behavior of SMA is necessary to improve their safety. To this aim an accurate evaluation of the maximum stress level under cyclic loading of alternating sign becomes highly important.

The problem of bending of a SMA beam was investigated numerically by many authors by means of finite element simulation, e.g. Auricchio and Sacco (1997), Auricchio et al. (2011), and Poorasadion, et al. (2015) or by numerical procedures based on the partition of the beam cross-section into a number of thin layers (De la Flor et al., 2011). Analytical approaches have been also tempted by some authors, but they are limited to a loading-unloading cycle and have not been extend to reversed loading. In 
particular, Mirzaeifar et al. (2013) provided explicit expressions for the stress and martensitic volume fraction distributions both in circular and rectangular cross sections upon a superelastic loadingunloading cycle and used these expressions for obtaining closed-form relations between bending moment and curvature. They also took into account for the tension-compression asymmetry on the bending response of SMAs and compared the theoretical predictions with the results of three-point bending tests on an SMA beam and finite element investigations. Ostadrahimi et al. (2015) presented an analytical solution for pure bending of SMA beams with rectangular cross section as well as symmetric behavior in tension and compression at high (pseudo-elasticity) and low (shape memory effect) temperatures. They assumed the same Young's modulus for both phases, thus obtaining piecewise linear stress variation within the cross section. Eshghinejad and Elahinia (2015) also derived an analytical expression for the bending moment in a superelastic SMA beam loaded at the tip and evaluated its deflection by assuming linear stress-strain relations in all regions and using a semi-analytical approach. Viet et al. (2018) worked out an analytical solution for the problem of SMA cantilever beams subjected to tip load throughout a full loading-unloading cycle. The analysis was based on Timoshenko beam theory and was later extended to account for tensile-compressive asymmetry in SMA response (Viet et al., 2019). The asymmetric tension-compression behavior of the SMA has been observed and modelled under direct bending by Rejzner et al. (2002), Fahimi et al., (2019), and Viet et al. (2019).

Most of these analyses investigate the superelastic behavior of SMA beams in order to exploit the self-centering mechanism, and thus they are restricted to temperatures higher than the start temperature of the austenitic transformation $A_{s}$. In this case, the reverse austenitic transformation is completed before the complete unloading. Therefore, these works do not take into consideration the transformation between the two Martensite variants, which may occur during unloading and reversed loading at temperature lower than $A_{s}$. The shape memory effect of SMA beams, which is essential for application to dampers and dissipating devices, is indeed observed under cyclic loading at temperature lower than the start temperature of the austenitic transformation $A_{s}$, e.g. for the NiTinol alloy $\left(\mathrm{Ni}_{55} \mathrm{Ti}\right)$ considered by Brinson (1993) this effect occurs at room temperature. In this case, during unloading the axial stress changes its sign in some parts of the beam cross section and it may trigger the Martensite reorientation process. Such transformation then continues under reversed bending. However, no complete and detailed analytical study of a SMA beam under reversed bending has been performed up to now. Recently, Radi (2020) performed an analytical investigation of the shape memory effect in SMA circular rod under cyclic torsional loading by adopting a 3D SMA constitutive model that incorporates two opposite Martensite variants. He also analyzed the transformation and reorientation processes into multiple martensitic variants in a previous work on 
the response of SMA thick-walled cylinders under internal pressure (Radi, 2018).

The present study extends the previous investigations to pure bending of SMA beams with rectangular cross section. In particular, closed-form solutions are obtained here for the axial stress and distribution of Martensite volume fractions at each step of a cyclic bending process of alternating sign, performed at constant temperature $T$ lower than $A_{s}$. These analytical results are then used for calculating the moment-curvature relation for a SMA beam cross section subjected to direct and reversed bending. This achievement is possible because a simple 1D phenomenological SMA constitutive model is adopted here, which assumes a linear evolution law with the axial stress for both Martensite variants. The simplifications adopted here preserve the basic features of SMA materials and they are required for achieving a closed form solution to the problem of reversed pure bending of SMA beams. Obviously, more refined constitutive models have been proposed in the last years. However, they necessarily require the adoption of numerical procedures, also for simulating simple mechanical problems. The constitutive model developed here originates from those proposed for multi-variants Martensite (Brinson, 1993; Brinson and Huang, 1996; Govindjee and Kasper, 1997, 1999; De la Flor et al., 2011; Marfia and Rizzoni, 2013; Rizzoni et al., 2013; Rizzoni and Marfia, 2015; Fahimi et al., 2019) and recently adopted by Radi (2020) for modelling cyclic torsional loading. It is able to simulate the Martensite reorientation process occurring when a transition from tensile to compressive stress, or vice versa, takes place. Here, the Martensite reorientation process under reverse bending is taken into consideration analytically for the first time.

The constitutive model is the 1D reduction of a 3D model already used in Radi (2018). It is sufficiently accurate for the present analysis because the stress field under pure bending is 1D. An equivalent reduction is performed also in other investigations of the pseudoelastic effect in SMA beam under bending, e.g. in Mirzaeifar et al. (2012), Ostadrahimi et al. (2015), Viet et al. (2018, 2019). Obviously, a 3D strain field arises in the beam so that the beam cross section can change his shape by expanding in the compressed zone and contracting in the tensile one, due also to the martensitic transformation and reorientation. This effect can be explicitly considered in the present analysis by specifying the strain components in the plane of the cross section under 1D stress field. However, the analysis has been focused here on the derivation of the analytical relation between bending moment and curvature during the full cyclic process taking into account for martensitic reorientation, rather than focusing on the cross section deformation.

Since no variation of temperature due to the thermal-mechanical coupling of SMA has been considered, then the isothermal solutions found here holds for very slow loading-unloading rates only. The fully coupled thermal-mechanical behaviors of SMA beams subjected to cyclic loading conditions is generally very complicated to be investigated analytically. Due to the complex heat 
diffusion process it would necessary require the adoption of a purely numerical procedure. This paper is organized as follows. The 1D constitutive model for SMA beams under pure bending is presented in Section 2. Different elastic Young's moduli are considered for the two phases and the elastic behavior of the SMA is assumed to depend on the volume fraction of Austenite and Martensite according to the Reuss scheme for the elastic response of a composite. Moreover, the model can simulate the martensitic reorientation process during unloading and reversed bending. Closed form solutions for the axial stresses and Martensite fractions within the cross section at each step of the first loading-unloading process are presented in Section 3, together with the corresponding relation between the applied bending moment and the beam curvature obtained by integrating the contribution of the axial stress within the cross-section. Similar closed form solutions are obtained in Section 4 for the reversed bending and subsequent elastic unloading. The results are then plotted in Section 5 and the effects of the constitutive parameters on the distribution of axial stresses and Martensite volume fractions within the cross-section are discussed therein. The analytical results are then validated against some analytical results for a loading-unloading cycle provided in the technical literature. Unfortunately, neither analytical nor experimental results can be found for the Martensite reorientation process under reversed bending loading. Conclusions are then drawn in Section 6. With respect to previous similar works, the fully analytical solution presented here significantly extends the range of validity to a full cycle of alternating sign of the applied bending moment. The accurate knowledge of the full stress field during the complete process is indeed the only way to ensure good limitation of the stress level for the safe and accurate design of SMA devices under bending.

\section{1D constitutive model for a SMA beam under pure bending}

Let $x$ and $y$ denote the principal centroidal axes of the rectangular cross section of a prismatic beam having width $b$ and height $2 a$. According to the classical Euler-Bernoulli theory, the axial strain $\varepsilon$ under pure bending is proportional to the beam curvature $\chi$ and is a linear function of the distance $y$ from the neutral axis of the cross section, which coincides with the $x$-axis, namely

$$
\varepsilon=\chi y, \quad \text { for }-a \leq y \leq a .
$$

By assuming tension-compression symmetry for simplicity, a symmetric distribution of the axial stress $\sigma$ along the height is expected, so that the neutral fibre always coincides with the centroidal $x$ axis. Therefore, reference is made only to the upper half of the cross section $(y \geq 0)$ in the following. The bending moment $M_{x}$ applied to the beam cross section can be calculated by taking the moment about the neutral axis of the stress distribution along the height, namely 


$$
M_{x}=2 b \int_{0}^{a} \sigma y d y
$$

According to Govindjee and Kasper $(1997,1999)$ and Rizzoni and Marfia (2015), let $\xi^{+}$and $\xi^{-}$denote the volume fractions of Martensite stretched in the positive (tensile) and negative (compressive) directions, respectively. Then, the effective volume fraction of Martensite $\xi$ is defined by the difference $\xi=\xi^{+}-\xi^{-}$and the total volume fraction of Martensite is given by the sum $\xi_{\text {tot }}=\xi^{+}+\xi^{-}$, where $-1 \leq \xi \leq 1$ and $0 \leq \xi_{\text {tot }} \leq 1$. Moreover, the 1D rate constitutive relationships between the axial stress $\sigma$, the axial strain $\varepsilon$ in (2.1) and the effective volume fraction of Martensite $\xi$ are assumed in the following linear form

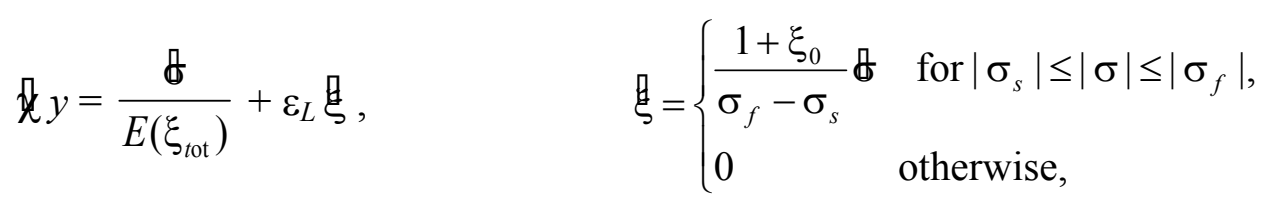

where $\varepsilon_{L}$ is the maximum transformation strain attained under uniaxial loading when the solid is composed of fully oriented Martensite, $\xi_{0}$ is the effective volume fraction Martensite at the beginning of each transformation, $E\left(\xi_{\text {tot }}\right)$ is the Young's modulus of the SMA material, which is assumed to depend on the total Martensite volume fraction according to the Reuss scheme for the elastic response of a composite, namely

$$
\frac{1}{E\left(\xi_{\text {tot }}\right)}=\frac{1-\xi_{\text {tot }}}{E_{\mathrm{A}}}+\frac{\xi_{\text {tot }}}{E_{\mathrm{M}}},
$$

being $E_{\mathrm{A}}$ and $E_{\mathrm{M}}$ the Young's moduli of the two phases. In eqn $(2.3)_{2}, \sigma_{s}$ and $\sigma_{f}$ denote the critical axial stresses for the start and finish martensitic transformations at temperature $T$, respectively, and they are defined by

$$
\sigma_{s}=\sigma_{s}^{c r}+C_{\mathrm{M}}\left(T-M_{s}\right), \quad \sigma_{f}=\sigma_{f}^{c r}+C_{\mathrm{M}}\left(T-M_{s}\right),
$$

where $C_{\mathrm{M}}$ is the slope of the martensitic transformation lines in the uniaxial stress-temperature phase diagram sketched in Fig. 1, and $\sigma_{s}^{c r}$ and $\sigma_{f}^{c r}$ are the uniaxial critical stresses for the start and finish of the martensitic transformation at temperature $M_{S}$ (Brinson and Huang, 1996).

Before loading, the SMA beam is assumed in the austenitic phase, so that $\xi=\xi_{\text {tot }}=\xi_{0}=0$. As the beam is loaded in bending, a single Martensite variant is produced in the upper half of the cross section, according to the integrated form of the evolution law $(2.3)_{2}$ 


$$
\xi=\xi^{+}= \begin{cases}0, & \text { for } \quad \sigma \leq \sigma_{s}, \\ \frac{\sigma-\sigma_{s}}{\sigma_{f}-\sigma_{s}}, & \text { for } \quad \sigma \leq \sigma \leq \sigma_{f}, \\ 1, & \text { for } \quad \sigma \geq \sigma_{f} .\end{cases}
$$

During unloading and reversed bending, the effective and total Martensite volume fractions follows from the integration of the linear evolution law $(2.3)_{2}$ as

$$
\xi=\xi^{+}-\xi^{-}= \begin{cases}\xi_{0}, & \text { for } \sigma \geq-\sigma_{s}, \\ \xi_{0}+\left(1+\xi_{0}\right) \frac{\sigma+\sigma_{s}}{\sigma_{f}-\sigma_{s}}, & \text { for }-\sigma_{f} \leq \sigma \leq-\sigma_{s}, \\ -1, & \text { for } \sigma \leq-\sigma_{f},\end{cases}
$$

and

$$
\xi_{\mathrm{tot}}=\xi^{+}+\xi^{-}=\left\{\begin{array}{lc}
\xi_{0}, & \text { for } \quad \sigma \geq-\sigma_{s} \\
1-\left(1-\xi_{0}\right) \frac{\sigma+\sigma_{f}}{\sigma_{f}-\sigma_{s}}, & \text { for } \quad-\sigma_{f} \leq \sigma \leq-\sigma_{s} \\
1, & \text { for } \quad \sigma \leq-\sigma_{f}
\end{array}\right.
$$

respectively, where $\xi_{0}$ is the effective volume fraction Martensite at the beginning of each stage. Eqns (2.7) and (2.8) agree with the 1D constitutive relations for the multivariant Martensite volume fractions considered by Govindjee and $\operatorname{Kasper}(1997,1999)$, De la Flor (2011), Rizzoni et al. (2012) and Radi (2020).

\section{Loading-unloading cycle under pure bending}

In the initial state of Austenite, the beam displays linear elastic behavior and thus the axial stress within the cross section is given by the following linear function of the beam curvature

$$
\frac{\sigma}{\sigma_{s}}=\kappa \frac{y}{a}, \quad \text { for } 0 \leq \kappa \leq 1,
$$

where

$$
\kappa=\frac{E_{\mathrm{A}}}{\sigma_{s}} \chi a,
$$

is the normalized beam curvature. Consequently, the martensitic transformation starts at the top of the cross section, namely at $y=a$, when the axial stress attains the critical stress $\sigma_{s}$ therein, namely 
for $M_{x}=M_{s}$, where

$$
M_{s}=\frac{2}{3} b a^{2} \sigma_{s}
$$

Let

$$
\mu=\frac{M_{x}}{M_{s}}=\frac{3}{a^{2}} \int_{0}^{a} \frac{\sigma}{\sigma_{s}} y d y
$$

denotes the normalized bending moment applied to the cross section, then the following normalized linear relation holds between bending moment and beam curvature under pure elastic loading

$$
\mu=\kappa, \quad \text { for } 0 \leq \kappa \leq 1 .
$$

If the bending moment is increased, then the martensitic transformation takes place at the upper part of the cross section, namely at $y_{s} \leq y \leq a$, where

$$
y_{s}=\frac{a}{\kappa},
$$

and it propagates inward. According to eqns (2.3) and (2.4), the stress and beam curvature in the transforming region are related by the linear rate relation

$$
\frac{\sigma_{s}}{E_{\mathrm{A}}} \frac{y}{a} \mathbb{k}=\left(\frac{1-\xi}{E_{\mathrm{A}}}+\frac{\xi}{E_{\mathrm{M}}}+\frac{\varepsilon_{L}}{\sigma_{f}-\sigma_{s}}\right) \&, \quad \text { for }-y_{s} \leq y \leq y_{s},
$$

which can be integrated starting from the occurring of the martensitic transformation at height $y$, by using eqn (2.6), namely

$$
\kappa \frac{y}{a}-1=\frac{1}{\sigma_{s}} \int_{\sigma_{s}}^{\sigma}\left(1+\delta-\frac{\Gamma}{\beta}+\frac{\Gamma}{\beta} \frac{\sigma}{\sigma_{s}}\right) d \sigma, \quad \text { for } y_{s} \leq y \leq a,
$$

where the following non-dimensional parameters have been introduced

$$
\beta=\frac{\sigma_{f}-\sigma_{s}}{\sigma_{s}}, \quad \delta=\frac{\varepsilon_{L} E_{\mathrm{A}}}{\sigma_{f}-\sigma_{s}}, \quad \Gamma=\frac{E_{\mathrm{A}}-E_{\mathrm{M}}}{E_{\mathrm{M}}} .
$$

The integration of eqn (3.8) then provides a quadratic equation for the axial stress $\sigma$

$$
\left(1+\delta-\frac{\Gamma}{\beta}\right)\left(\frac{\sigma}{\sigma_{s}}-1\right)+\frac{\Gamma}{2 \beta}\left(\frac{\sigma^{2}}{\sigma_{s}^{2}}-1\right)=\kappa \frac{y}{a}-1, \quad \text { for } y_{s} \leq y \leq a,
$$

which admits the following solution for the axial stress within the cross section

$$
\frac{\sigma}{\sigma_{s}}= \begin{cases}\kappa \frac{y}{a}, & \text { for } 0 \leq y \leq y_{s}, \\ 1+B\left[\sqrt{1+\frac{2}{B_{1}}\left(\kappa \frac{y}{a}-1\right)}-1\right], & \text { for } y_{s} \leq y \leq a,\end{cases}
$$

where 


$$
B=\frac{\beta}{\Gamma}(1+\delta), \quad B_{1}=\frac{\beta}{\Gamma}(1+\delta)^{2}
$$

According to eqns (2.6) and (4.10), the distribution of the Martensite volume fraction within the cross section is given by

$$
\xi= \begin{cases}0, & \text { for } 0 \leq y \leq y_{s}, \\ \frac{B}{\beta}\left[\sqrt{1+\frac{2}{B_{1}}\left(\kappa \frac{y}{a}-1\right)}-1\right], & \text { for } \quad y_{s} \leq y \leq a .\end{cases}
$$

Both the axial stress and the volume fraction of Martensite display a nonlinear variation with the $y$ coordinate in the transforming region for $y_{s} \leq y \leq a$, which becomes linear for $\Gamma=0$, namely for equal Young's moduli of the two phases.

A further increment of the bending moment applied to the cross section then causes the occurring of the complete martensitic transformation at $y=a$, where $\xi=1$. Correspondingly, the normalized beam curvature and the height of start martensitic transformation are given by

$$
\kappa_{1}=1+\left(\frac{\Gamma}{2}+1+\delta\right) \beta, \quad y_{1}=\frac{a}{\kappa_{1}},
$$

as they follow from eqns (3.12) and (3.6), respectively. The introduction of the axial stress (4.10) in the balance condition (3.4) 1 then yields the normalized moment-curvature relation, which holds during this stage of loading

$$
\mu(\kappa)=\frac{1}{5 \kappa^{2}}\left[5+B B_{1}\left(B_{1}-5\right)-\frac{15}{2}(B-1)\left(\kappa^{2}-1\right)-\frac{B}{\sqrt{B_{1}}}\left(B_{1}-2-3 \kappa\right)\left(B_{1}-2+2 \kappa\right)^{3 / 2}\right],
$$

namely for $1 \leq \kappa \leq \kappa \kappa_{1}$.

The axial stress at $y=a$ attains the critical value $\sigma_{f}$ under the normalized bending moment $\mu_{1}=\mu\left(\kappa_{1}\right)$. If the bending moment is increased, then the fully transformed region propagates inwards. Within this region, the rate eqn (3.7) can be integrated starting from the occurring of the complete martensitic transformation at height $y$, by using the conditions $\xi=1$, thus obtaining

$$
\frac{\sigma-\sigma_{f}}{\sigma_{s}}=\frac{1}{1+\Gamma}\left(\kappa \frac{y}{a}-\kappa_{1}\right), \quad \text { for } y_{f} \leq y \leq a,
$$

where the height

$$
y_{f}=\frac{\kappa_{1}}{\kappa} a,
$$

denotes the front of finish martensitic transformation, where $\sigma=\sigma_{f}$.

During the last stage of the loading process, the cross section is partitioned into an inner purely austenitic inner region for $|y| \leq y_{s}$, an intermediate transforming region for $y_{s} \leq|y| \leq y_{f}$, and an outer 
purely martensitic region for $y_{f} \leq|y| \leq a$. From eqn (4.10), (3.12), and (3.15), the corresponding distributions of the axial stress and Martensite volume fraction within the upper half of the cross section are given by

$$
\frac{\sigma}{\sigma_{s}}= \begin{cases}\kappa \frac{y}{a} & \text { for } 0 \leq y \leq y_{s}, \\ 1+B\left[\sqrt{1+\frac{2}{B_{1}}\left(\kappa \frac{y}{a}-1\right)}-1\right] & \text { for } y_{s} \leq y \leq y_{f}, \\ 1+\beta+\frac{1}{1+\Gamma}\left(\kappa \frac{y}{a}-\kappa_{1}\right) & \text { for } y_{f} \leq y \leq a,\end{cases}
$$

and

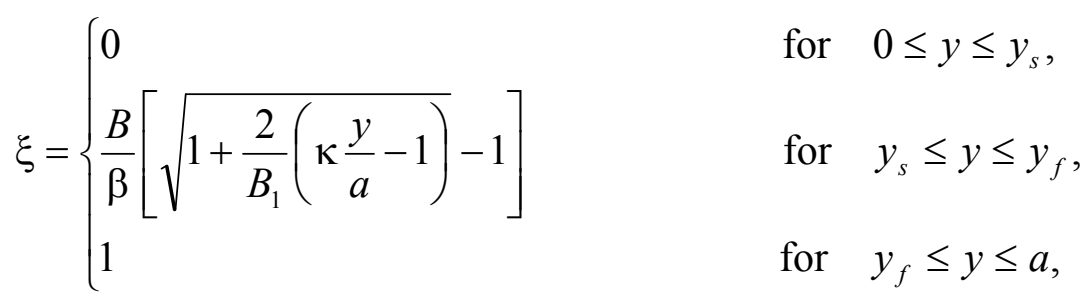

respectively. Therefore, during the loading process both the axial stress and the Martensite volume fraction vary linearly with $y$ across the inner austenitic and the outer martensitic regions, and display a weak nonlinear variation within the intermediate transforming region.

During the last stage of the loading process, the normalized bending moment $\mu$ then follows from $(3.4)_{1}$ and (3.17) as a nonlinear function of the normalized beam curvature $\kappa$

$$
\mu(\kappa)=\frac{C}{\kappa^{2}}+\frac{\kappa}{1+\Gamma}+\frac{3}{2}\left(1+\beta-\frac{\kappa_{1}}{1+\Gamma}\right), \quad \text { for } \kappa_{1} \leq \kappa \leq \kappa_{\max },
$$

where

$$
\begin{aligned}
C= & \frac{\kappa_{1}^{3}}{2(1+\Gamma)}-\frac{1}{2}\left[1-3 B+3(B+\beta) \kappa_{1}^{2}\right]-\frac{B}{5} \sqrt{1+\frac{2}{B_{1}}\left(\kappa_{1}-1\right)}\left(B_{1}-3 k_{1}-2\right)\left(B_{1}+2 k_{1}-2\right) \\
& +\frac{B}{5} B_{1}\left(B_{1}-5\right),
\end{aligned}
$$

Note that for equal Young's moduli, namely for $\Gamma=0$, the distributions of axial stress (3.17) and effective Martensite volume fraction (3.18) within each region of the upper half of the cross section become linear in $y$, in agreement with the findings of Ostadrahimi et al. (2015), namely 


$$
\frac{\sigma}{\sigma_{s}}= \begin{cases}\frac{y}{a} \kappa, & \text { for } 0 \leq y \leq y_{s}, \\ 1+\frac{1}{1+\delta}\left(\frac{y}{a} \kappa-1\right), & \text { for } y_{s} \leq y \leq y_{f}, \\ 1+\beta+\left(\frac{y}{a} \kappa-\kappa_{1}\right), & \text { for } y_{f} \leq y \leq a,\end{cases}
$$

and

$$
\xi= \begin{cases}0, & \text { for } 0 \leq y \leq y_{s}, \\ \frac{1}{\beta(1+\delta)}\left(\frac{y}{a} \kappa-1\right), & \text { for } y_{s} \leq y \leq y_{f}, \\ 1, & \text { for } y_{f} \leq y \leq a,\end{cases}
$$

where $y_{s}$ and $y_{f}$ are given by (3.6) and (3.16) calculated for $\Gamma=0$.

Let $\kappa_{\max }=a / y_{s}$ and $\mu_{\max }=\mu\left(\kappa_{\max }\right)$ denote the normalized beam curvature at the end of the loading process and the corresponding normalized bending moment, respectively. Moreover, let $\sigma_{\max }$ and $\xi_{\max }$ denote the distributions along the height of the beam cross section of axial stress and volume fraction of Martensite at the same stage, which follow from (3.17) and (3.18) for $\kappa=\kappa_{\max }$, respectively.

\subsection{Unloading}

The Martensite distribution does not vary during elastic unloading as well as the heights $y_{s}$ and $y_{f}$, which are given by (3.6) and (3.16) for $\kappa=\kappa_{\max }$. The integration of the rate constitutive equation (2.3) for $\xi=\xi_{\max }(y)$, and thus $\xi=0$, by using the Reuss scheme (2.4), then yields the axial stress during elastic unloading as a function of the normalized curvature

$$
\frac{\sigma}{\sigma_{s}}=\frac{\sigma_{\max }}{\sigma_{s}}-\frac{\kappa_{\max }-\kappa}{1+\Gamma \xi_{\max }} \frac{y}{a}, \quad \text { for } 0 \leq y \leq a .
$$

The corresponding moment-curvature relation follows from the introduction of the stress field (3.23) in the balance condition $(3.4)_{1}$

$$
\mu=\mu_{\max }-\left(\kappa_{\max }-\kappa\right) \frac{3}{a^{3}} \int_{0}^{a} \frac{y^{2}}{1+\Gamma \xi_{\max }} d y .
$$

The change of variable $t=1+\Gamma \xi_{\max }(y)$ in the integral in eqn (3.24), by using (3.18) for $\xi_{\max }(y)$, then gives

$$
\mu=\mu_{\max }-\left(\kappa_{\max }-\kappa\right) m,
$$

where 


$$
m=\frac{1}{\kappa_{\max }^{3}}\left\{1+\frac{\kappa_{\max }^{3}-\kappa_{1}^{3}}{1+\Gamma}+\frac{3 \beta^{3}}{4 \Gamma^{3}} \int_{1}^{1+\Gamma}\left[(t+\delta)^{2}-(1+\delta)^{2}+2 \frac{\Gamma}{\beta}\right]^{2}\left(1+\frac{\delta}{t}\right) d t\right\},
$$

and the integral in (3.26) is calculated analytically in Appendix A. Note that $m \rightarrow 1$ as $\Gamma \rightarrow 0$.

The elastic incremental behavior of the bar ends when the axial stress (3.23) reaches the negative critical stress $-\sigma_{s}$ at $y=y_{f}$, where $\xi_{\max }=1$ and $\sigma_{\max }=\sigma_{f}$, namely for $\kappa=\kappa_{2}$ and $\mu=\mu_{2}$, where

$$
\kappa_{2}=\kappa_{\max }-(1+\Gamma)(2+\beta) \frac{\kappa_{\max }}{\kappa_{1}}, \quad \quad \mu_{2}=\mu_{\max }-(1+\Gamma)(2+\beta) \frac{\kappa_{\max }}{\kappa_{1}} m .
$$

Indeed, Martensite reorientation takes place before the complete unloading of the cross section if $\mu_{2}>$ 0 , namely if the maximum beam curvature $\kappa_{\max }$ is larger than a limit value $\kappa_{\text {lim }}$ given by the largest positive real root of the following cubic equation obtained from the introduction of eqns (3.19) and $(3.26)$ in $(3.27)_{2}$ :

$$
\left(\frac{1}{1+\Gamma}-\frac{2+\beta}{\kappa_{1}}\right) \kappa_{\lim }^{3}+\frac{3}{2}\left(1+\beta-\frac{\kappa_{1}}{1+\Gamma}\right) \kappa_{\lim }^{2}=(2+\beta)\left[\frac{1+\Gamma}{\kappa_{1}}\left(1+\frac{3 \beta^{3}}{4 \Gamma^{3}} I\right)-\kappa_{1}^{2}\right]-C,
$$

where the constant $C$ is defined in (3.20) and $I$ denotes the integral calculated in Appendix A.

If $\kappa_{\max }<\kappa_{\text {lim }}$ then the unloading process is entirely elastic and when the bending moment is completely released the residual beam curvature is

$$
\kappa_{\text {res }}=\kappa_{\max }-\mu_{\max } / m \text {. }
$$

Conversely, if $\kappa_{\max }>\kappa_{\lim }$ then Martensite reorientation takes place before complete unloading according to eqn (2.7) for $\xi_{0}=\xi_{\max }$. In this case, two new regions where Martensite reorientation occurs spread out from the boundary between the intermediate transforming regions and the fully martensitic outer regions at $y=y_{f}$. Let us denote with $y^{\prime}$ and $y^{\prime \prime}$ the lower and upper heights delimiting these new regions, with $y^{\prime} \leq y_{f} \leq y^{\prime \prime}$, which are defined by the condition that the axial stress at $y=y^{\prime}$ and $y=y^{\prime \prime}$ must coincide with the negative critical stress $-\sigma_{s}$. Therefore, the following two relations between $\kappa, y^{\prime}$ and $y^{\prime \prime}$ follow from eqn (3.23)

$$
\sigma_{\max }\left(y^{\prime}\right)-\sigma_{s} \frac{\kappa_{\max }-\kappa}{1+\Gamma \xi_{\max }\left(y^{\prime}\right)} \frac{y^{\prime}}{a}=-\sigma_{s}, \quad \sigma_{\max }\left(y^{\prime \prime}\right)-\sigma_{s} \frac{\kappa_{\max }-\kappa}{1+\Gamma} \frac{y^{\prime \prime}}{a}=-\sigma_{s} .
$$

The introduction of eqn (3.21) for $\sigma_{\max }\left(y^{\prime}\right)$ with $\kappa=\kappa_{\max }$ in eqn (3.30) $)_{1}$ then yields the normalized beam curvature $\kappa$ as a function of the height $y^{\prime}$ :

$$
\kappa=\kappa_{\max }-\left[2+\beta \xi_{\max }\left(y^{\prime}\right)\right]\left[1+\Gamma \xi_{\max }\left(y^{\prime}\right)\right] \frac{R}{y^{\prime}}, \quad \text { for } y^{\prime} \leq y \leq y_{f} .
$$

By using eqn (3.22) for $\xi_{\max }\left(y^{\prime}\right)$ with $\kappa=\kappa_{\max }$, eqn (3.31) can be explicitly solved for $y^{\prime}$ as a function of the normalized beam curvature $\kappa$ 


$$
\frac{y^{\prime}}{a}=\left(\frac{1+2 \delta}{\Gamma}-\frac{2}{\beta}\right) \frac{(\delta \beta-2 \Gamma) \kappa_{\max }-(1+\delta) \kappa \beta}{\left(\kappa_{\max }+\kappa\right)^{2}}\left\{1-\sqrt{1-\frac{2 \beta \Gamma\left(\kappa_{\max }+\kappa\right)^{2}}{\left[(\delta \beta-2 \Gamma) \kappa_{\max }-(1+\delta) \beta \kappa\right]^{2}}}\right\},
$$

where $y_{s} \leq y^{\prime} \leq y_{f}$. In the same way, the introduction in eqn $(3.30)_{2}$ of the stress field $\sigma_{\max }\left(y^{\prime \prime}\right)$ obtained from (3.17) for $\kappa=\kappa_{\max }$ then yields the height $y^{\prime \prime}$ as a function of $\kappa$

$$
\frac{y^{\prime \prime}}{a}=\frac{1}{\kappa}\left[\kappa_{1}-(1+\Gamma)(\beta+2)\right],
$$

where $y_{f} \leq y^{\prime \prime} \leq a$. Let us consider a material point at height $y$ laying between $y^{\prime}$ and $y_{f}$. According to eqn (3.31), the reorientation of the primary Martensite variant into the opposite one starts there when $\kappa=\kappa_{y}$, where

$$
\kappa_{y}=\kappa_{\max }-\left[2+\beta \xi_{\max }(y)\right]\left[1+\Gamma \xi_{\max }(y)\right] \frac{a}{y}, \quad \text { for } y^{\prime} \leq y \leq y_{f} .
$$

Therefore, by subtracting eqn (3.34) from eqn (3.31) one has

$$
\kappa_{y}-\kappa=\left[2+\beta \xi_{\max }\left(y^{\prime}\right)\right]\left[1+\Gamma \xi_{\max }\left(y^{\prime}\right)\right] \frac{a}{y^{\prime}}-\left[2+\beta \xi_{\max }(y)\right]\left[1+\Gamma \xi_{\max }(y)\right] \frac{a}{y}, \text { for } y^{\prime} \leq y \leq y_{f}
$$

At the same time, for $\kappa=\kappa_{y}$, Martensite reorientation also starts at another material point at height $y$ ranging between $y_{f}$ and $y^{\prime \prime}$, and thus $\kappa_{y}$ must satisfy the following condition also

$$
\kappa_{y}=\left[\kappa_{1}-(1+\Gamma)(\beta+2)\right] \frac{a}{y}, \quad \text { for } y_{f} \leq y \leq y^{\prime \prime},
$$

according to (3.33). Then, eqns (3.33) and (3.36) yield

$$
\kappa_{y}-\kappa=\left[\kappa_{1}-(1+\Gamma)(\beta+2)\right]\left(\frac{a}{y}-\frac{a}{y^{\prime \prime}}\right), \quad \text { for } y_{f} \leq y \leq y^{\prime \prime} .
$$

The introduction of relations (2.4) and (3.9) in the rate constitutive eqns (2.3) then yields the following rate equation,

$$
E_{\mathrm{A}} y \mathbb{R}=\left[1+\xi_{\text {tot }} \Gamma+\delta\left(1+\xi_{\max }\right)\right] \&, \quad \text { for } y^{\prime} \leq y \leq y^{\prime \prime},
$$

which holds for a material point at coordinate $y$ experiencing the reorientation of the Martensite variant. Integration of the rate constitutive equation (3.38) from the start of the martensitic reorientation at height $y$, by using relations (2.8) for $\xi_{\text {tot }}$ with $\xi_{0}=\xi_{\max }$, then gives

$$
\frac{y}{a}\left(\kappa-\kappa_{y}\right)=\frac{1}{\sigma_{s}} \int_{-\tau_{s}}^{\tau}\left[1+\Gamma+\delta\left(1+\xi_{\max }\right)-\Gamma\left(1-\xi_{\max }\right) \frac{\sigma+\sigma_{f}}{\sigma_{f}-\sigma_{s}}\right] \mathrm{d} \sigma, \quad \text { for } y^{\prime} \leq y \leq y^{\prime \prime},
$$

being $-\sigma_{s}, \kappa_{y}$ and $\xi_{\max }$ the axial stress, the normalized beam curvature and the total volume fraction of Martensite at the beginning of the reorientation process, respectively. The calculation of the definite integral in (3.39) then yields the following quadratic equation for the axial stress $\sigma$ during the Martensite reorientation process 


$$
\frac{\sigma^{2}}{\sigma_{s}^{2}}+2\left[1-\beta \frac{1+\delta+(\delta-\Gamma) \xi_{\max }}{\Gamma\left(1-\xi_{\max }\right)}\right]\left(\frac{\sigma}{\sigma_{s}}+1\right)-1-\frac{2 \beta}{\Gamma}\left(\frac{\kappa_{y}-\kappa}{1-\xi_{\max }}\right) \frac{y}{a}=0, \text { for } y^{\prime} \leq y \leq y_{f},
$$

Therefore, the admissible solution of eqn (3.40) for $0<\xi_{\max }<1$ is

$$
\frac{\sigma}{\sigma_{s}}=-1-\beta \frac{1+\delta+(\delta+\Gamma) \xi_{\max }}{\Gamma\left(1-\xi_{\max }\right)}\left\{\sqrt{1+\frac{2 \Gamma\left(1-\xi_{\max }\right)\left(\kappa_{y}-\kappa\right)}{\beta\left[1+\delta+(\delta+\Gamma) \xi_{\max }\right]^{2}} \frac{y}{a}}-1\right\}, \quad \text { for } y^{\prime} \leq y \leq y_{f} .
$$

The terms $\kappa_{y}-\kappa$ and $y^{\prime}$ follow from eqns (3.35) and (3.32), respectively, in terms of the beam curvature $\kappa$.

For $\xi_{\max }=1$, eqn (3.39) provides the following result for the axial stress

$$
\frac{\sigma}{\sigma_{s}}=-1-\frac{\kappa_{y}-\kappa}{1+\Gamma+2 \delta} \frac{y}{a}, \quad \text { for } y_{f} \leq y \leq y^{\prime \prime}
$$

where the quantities $\kappa_{y}-\kappa$ and $y^{\prime \prime}$ are given in eqns (3.37) and (3.33) in terms of $\kappa$.

During this stage, the rest of the cross section displays only elastic behavior. Therein, the axial stress is thus given by

$$
\frac{\sigma}{\sigma_{s}}=\frac{\sigma_{\max }}{\sigma_{s}}-\frac{\kappa_{\max }-\kappa}{1+\Gamma \xi_{\max }} \frac{y}{a}, \quad \text { for } 0 \leq y \leq y^{\prime} \text { and } y^{\prime \prime} \leq y \leq a .
$$

The distribution of effective Martensite in the regions where the reorientation of the Martensite variant occurs then follows from eqn (2.7) for $\xi_{0}=\xi_{\max }$ as

$$
\xi=\xi_{\max }+\frac{1+\xi_{\max }}{\beta}\left(\frac{\sigma}{\sigma_{s}}+1\right), \quad \text { for } y^{\prime} \leq y \leq y^{\prime \prime} .
$$

where the ratio $\sigma / \sigma_{\mathrm{s}}$ follows from eqn (3.41) for $y^{\prime} \leq y \leq y_{f}$ or eqn (3.42) for $y_{f} \leq y \leq y^{\prime \prime}$. In the rest of the cross section, the volume fraction of Martensite is still equal to $\xi_{\max }$.

Therefore, the axial stress and Martensite volume fraction display linear distribution along the height of the cross section in the inner austenitic region at $y \leq y_{s}$ only, according to eqns (3.43) and (3.44) for $\xi_{\max }=0$, whereas their distributions in the rest of the cross section are clearly not linear.

As the bending moment is decreased or applied with the opposite sign, the boundaries $y^{\prime}$ and $y^{\prime \prime}$ moves inward and outward, respectively, and thus the boundary $y^{\prime \prime}$ may reach the outer surface when $y^{\prime \prime}=a$, namely for $\sigma(a)=-\sigma_{s}$. According to eqn (3.33), this condition occurs for $\kappa=\kappa_{3}$, where

$$
\kappa_{3}=\kappa_{1}-(1+\Gamma)(\beta+2), \quad \text { for } y_{f} \leq y^{\prime \prime} \leq a .
$$

During this stage, the axial stress and Martensite distributions are still provided by eqns (3.41)-(3.44). The introduction of the axial stress (3.41)-(3.43) in the balance condition $(3.4)_{1}$ then provides the normalized moment-curvature relation within the range $\kappa_{3} \leq \kappa \leq \kappa_{2}$. In this case, the residual beam curvature when the bending moment is completely released is given by the solution of the 
transcendent equation $\mu\left(\kappa_{\text {res }}\right)=0$.

In the special case of equal Young's moduli of the two phases, namely for $\Gamma \rightarrow 0$, the axial stress in (3.41) assumes the simple expression

$$
\frac{\sigma}{\sigma_{s}}=-1-\frac{\kappa_{y}-\kappa}{1+\delta+\delta \xi_{\max }} \frac{y}{a}, \quad \text { for } y^{\prime} \leq y \leq y_{f}
$$

where $\xi_{\max }$ is given by (3.22) for $\kappa=\kappa_{\max }$.

\section{Reversed bending}

If an opposite bending moment is applied to the cross section after complete unloading and its magnitude is gradually increased, then the axial stress and Martensite distributions during this stage are still given by eqn (3.23) for $\kappa_{2} \leq \kappa \leq \kappa_{\text {res }}$ and by eqns (3.41)-(3.44) for $\kappa_{3} \leq \kappa<\kappa_{2}$. Indeed, eqns (3.41)-(3.44) hold true till the outer region fully made of the first variant Martensite disappears when $y^{\prime \prime}=a$ namely for $\kappa=\kappa_{3}$. Actually, these equations provide the axial stress and Martensite distributions also for $\kappa<\kappa_{3}$. In this case, however, eqn (3.33) yields $y^{\prime \prime}>a$. The introduction of the stress fields (3.41)-(3.43) in the balance condition (3.4) then provides the moment-curvature relation for this stage of reversed bending, which continues till one of the following two conditions is met. Namely, either the axial stress reaches the critical stress $-\sigma_{s}$ for the start of the Martensite reorientation at height $y^{\prime}$, i.e. for $y^{\prime}=y_{s}$, or the axial stress reaches the critical stress $-\sigma_{f}$ for the finish of the Martensite reorientation at the top of the cross section, i.e. for $y_{-f}=a$. According to (3.31), the former condition occurs for $\kappa=-\kappa_{\max }$, being $\xi_{\max }\left(y_{s}\right)=0$ and $\kappa_{\max }=a / y_{s}$. According to (3.42) and using relations (3.33), (3.37), and (3.13), the latter condition, namely $\sigma(a)=-\sigma_{f}$, occurs for $\kappa=\kappa_{4}$, where

$$
\kappa_{4}=-\kappa_{1}-\Gamma(\beta+2) .
$$

If the magnitude of the negative bending moment is further increased, then a region fully made of the negative Martensite variant originates at the top of the cross section and propagates inwards with height $y_{-f}$ defined by the condition $\sigma\left(y_{-f}\right)=-\sigma_{f}$, namely

$$
\frac{y_{-f}}{a}=-\frac{\kappa_{1}+(\beta+2) \Gamma}{\kappa}, \quad \text { for } \kappa<\kappa_{4},
$$

according to (3.36) and (3.42). In this case, the distributions of the axial stress and the effective Martensite volume fraction are given by eqns (3.43) and (3.18) for $0 \leq y \leq y^{\prime}$, by (3.41) and (3.44) for $y^{\prime} \leq y \leq y_{f}$, and by (3.42) and (3.44) for $y_{f} \leq y \leq y_{-f}$, respectively, where the heights $y^{\prime}$ and $y^{\prime \prime}$ are defined in (3.33) as functions of $\kappa$. Integration of eqn (2.3) in the outer fully martensitic region where $\xi=-1$, starting from the finish of martensitic transformation at height $y$, by using (4.1) and (4.2), then 
gives

$$
\frac{\sigma}{\sigma_{s}}=-1-\beta+\frac{1}{1+\Gamma}\left[\kappa_{1}+(\beta+2) \Gamma+\kappa \frac{y}{a}\right], \quad \text { for } y_{-f} \leq y \leq a .
$$

In both cases, the introduction of the axial stress fields in (3.4) then yields the corresponding bending moment-curvature relation.

Note from eqn (4.2) that for different Young's moduli, namely for $\Gamma>0$, then for $\kappa=-\kappa_{\max }$ one has $y^{\prime}=a \kappa_{1} / \kappa_{\max }=y_{f}<y_{-f}$. Therefore, the axial stress and Martensite distributions across the beam cross section for $\kappa=-\kappa_{\max }$ are not exactly opposite to those obtained from (3.21) and (3.22) for $\kappa=\kappa_{\max }$. On the contrary, for equal Young's moduli of the two phases, namely for $\Gamma=0$, then for $\kappa=-\kappa_{\max }$ one has $y^{\prime}=y_{f}$ and $y_{-f}=y_{f}$, namely the axial stress and Martensite distributions are exactly opposite to those obtained for $\kappa=\kappa_{\max }$.

The introduction of the axial stress fields (4-38), (3.42), and (3.43) or (4.3) in the balance condition $(3.4)_{1}$ then yields the bending moment-curvature relation for the last stage of the reversed bending process.

Let $\sigma_{\min }$ and $\xi_{\min }$ denote the distributions of axial stress and effective volume fraction of Martensite along the height of the beam cross section at the end of reversed bending for $\kappa_{\min }=-\kappa_{\max }$. They follow from (3.41)-(3.44) and (4.3), by using (3.13), (3.34), and (3.36), as

$$
\frac{\sigma_{\min }}{\sigma_{s}}=\left\{\begin{array}{ll}
-\frac{y}{a} \kappa_{\max }, & \text { for } 0 \leq y \leq y_{s}, \\
-1-\beta \frac{1+\delta+(\delta+\Gamma) \xi_{\max }}{\Gamma\left(1-\xi_{\max }\right)}\left\{\sqrt{1+2 \Gamma\left(1-\xi_{\max }\right) \frac{2 \kappa_{\max } y / a-\left(2+\beta \xi_{\max }\right)\left(1+\Gamma \xi_{\max }\right)}{\beta\left[1+\delta+(\delta+\Gamma) \xi_{\max }\right]^{2}}}-1\right.
\end{array}\right\},
$$

and

$$
\xi_{\min }= \begin{cases}0, & \text { for } 0 \leq y \leq y_{s}, \\ \xi_{\max }+\frac{1+\xi_{\max }}{\beta}\left(\frac{\sigma_{\min }}{\sigma_{s}}+1\right), & \text { for } y_{s} \leq y \leq \min \left\{y_{-f, \min }, a\right\}, \\ -1, & \text { for } \max \left\{y_{-f, \min }, a\right\} \leq y \leq a,\end{cases}
$$

where 


$$
y_{-f, \min }=\frac{\kappa_{1}+(\beta+2) \Gamma}{\kappa_{\max }} a .
$$

\subsection{Elastic unloading after reversed bending}

The integration of the rate constitutive equation (2.3) for $\frac{\theta}{=0}$, being $\xi=\xi_{\min }$, by using (2.4) for $\xi_{\text {tot }}=$ $\xi_{\max }$, then yields the axial stress during the subsequent elastic unloading process

$$
\frac{\sigma}{\sigma_{s}}=\frac{\sigma_{\min }}{\sigma_{s}}+\frac{\kappa_{\max }+\kappa}{1+\Gamma \xi_{\max }(y)} \frac{y}{a}, \quad \text { for } 0 \leq y \leq a .
$$

The corresponding applied bending moment is

$$
\mu=\mu_{\min }+\left(\kappa_{\max }+\kappa\right) m,
$$

according to (3.4) 1 and (4.7). The elastic unloading of the beam ends when $\mu=0$, for $\kappa=\kappa^{\prime}$ res where

$$
\kappa_{\text {res }}^{\prime}=-\kappa_{\max }-\mu_{\min } / m \text {. }
$$

The elastic behavior of the beam ends when the axial stress reaches the positive critical stress $\sigma_{s}$ at $y$ $=\min \left\{y_{-f, \min }, a\right\}$, namely for $\kappa=\kappa_{5}$ where

$$
\kappa_{5}= \begin{cases}-\frac{\kappa_{1}-\beta-2}{\kappa_{1}+(\beta+2) \Gamma} \kappa_{\max }, & \text { if } y_{-f, \min }<a, \\ -\frac{(1+\Gamma)(\beta \Gamma-2-8 \delta-2 \beta \delta)+4 \delta \kappa_{\max }}{2(1+\Gamma+2 \delta)}, & \text { if } a \leq y_{-f, \text { min }} .\end{cases}
$$

A further increase in the applied bending moment will trigger Martensite reorientation into the positive variant at height $y=\min \left\{y_{-f, \min }, a\right\}$.

\section{Results}

The results presented in the following make reference to the NiTinol constitutive parameters at room temperature $T=298^{\circ} \mathrm{K}$ considered by Brinson (1993), which are reported in Table 1 . The corresponding parameters defined in Sections 3 and 4 are $\beta=0.4575, \delta=64.1286$ and $\Gamma=1.5475$.

The distribution of the austenitic and martensitic phases varying the applied bending moment during loading are plotted in Fig. 2 together with the variations of the fronts of start and finish martensitic transformation, $y_{s}$ and $y_{f}$. This figure also provides the distribution of the two phases along a SMA cantilever beam loaded by a unit transversal force applied at the tip, where the bending moment increases linearly from the loaded tip to the built-in end.

The distributions of the axial stress and effective volume fraction of Martensite along the height of the upper-half cross section at each step of loading and reversed loading are plotted in Fig. 3 and 4, 
respectively, for $\mu_{\max }=2,3$, and 4 . These curves correspond to the end of the loading process for $\kappa$ $=\kappa_{\max }$, the end of elastic unloading for $\kappa=\kappa_{2}$, the complete unloading for $\kappa=\kappa_{\text {res, }}$, the reversed bending for $\kappa$ equal to $\kappa_{3}, 0, \kappa_{4}$, and $-\kappa_{\max }$, and the subsequent complete unloading for $\kappa=\kappa_{\text {res }}^{\prime}$.

The variation of the axial stress and Martensite volumetric fraction along the height of the cross section during first loading are piecewise linear in the inner fully austenitic region and in the outer fully martensitic region, whereas they are weakly non-linear in the intermediate transforming regions, since different Young's moduli are considered for the two phases. Note that in the last stage of loading (solid black lines), the axial stress significantly increases in the upper fully martensitic region, where the yield stress of the SMA material could be rapidly accomplished at the top surface, in agreement with the findings of Eshghinejad and Elahinia (2015).

During the subsequent elastic unloading, the axial stress decreases linearly with the height $y$ and no phase transformation or Martensite reorientation occur, till the negative critical stress $-\sigma_{f}$ is attained at $y_{f}$. This condition is accomplished for $\kappa=\kappa_{2}$ and $\mu=\mu_{2}$ defined in (3.27). Therefore, for $\kappa<\kappa_{2}$ the reorientation of the Martensite variant is triggered, starting from the region at height $y_{f}$. This process occurs during unloading only if the maximum bending moment applied to the cross section is larger than a limit value. For the Brinson SMA material at the considered room temperature, the (normalized) limit bending moment is $\mu_{\mathrm{lim}}=3.617$, this value is obtained by introducing the solution $\kappa_{\lim }=42.22$ of eqn (3.28) in eqn (3.19). The results plotted in Figs. $3 a$ and $3 b$ for $\mu_{\max }=2$ and 3, respectively, show indeed that the unloading process is entirely elastic and after complete unloading, namely for $\kappa=\kappa_{\text {res }}$ (dashed red lines), a wide region is subject to residual negative axial stress, whose magnitude however is not large enough for triggering Martensite reorientation. Conversely, the results obtained in Fig. $3 c$ for $\mu_{\max }=4>\mu_{\text {lim }}$ show that Martensite reorientation takes place at height $y_{f}$ during unloading and spreads inwards and outwards as the applied bending moment is further decreased. In this region, the axial stress $\sigma$ at the end of unloading displays a weak and limited nonlinear variation with the coordinate $y$, according to (3.41), being actually almost constant and a bit more negative than $-\sigma_{s}$ (red dashed line in Fig. $3 c$ for $\kappa=\kappa_{\text {res}}$ ). It may be also observed that at the end of unloading after first loading, the axial stress vanishes at five points within the cross section. The maximum residual axial stress is attained at the top surface and it increases remarkably with $\mu_{\max }$. For $\mu_{\max }<\mu_{\text {lim }}$ Martensite reorientation takes place during reversed bending, always starting from height $y_{f}$.

Under reversed bending, the axial stress tends to be negative and almost uniform in the upper half of the cross section till the negative critical stress $-\sigma_{f}$ is attained at the top for $\kappa=\kappa_{4}$ (see the lines for $\kappa$ equal to $\kappa_{3}, 0, \kappa_{4}$ in Fig. 3). However, if the magnitude of the applied negative bending is further 
increased, then the magnitude of the negative axial stress rapidly increases at the top of the cross section (see the dash-dotted blue lines for $\kappa=-\kappa_{\max }$ in Fig. 3). For different Young's moduli of the two phases, the distributions of the axial stress at the end of the reversed bending for $\kappa=-\kappa_{\max }$ (dashdotted blue lines) are not exactly opposite to those obtained for $\kappa=\kappa_{\max }$ (solid black lines). The axial stress is indeed opposite, but its magnitude is smaller than that observed at the end of direct loading. The distributions of residual axial stress at complete unloading subsequent to reversed bending, namely for $\kappa=\kappa^{\prime}$ res, are plotted in Fig. 3 as dotted black lines. Again, the axial stress vanishes at five points within the cross section, the maximum magnitude of the residual axial stress is attained at the top surface and it increases remarkably with $\mu_{\max }$. Note that for the values of $\mu_{\max }$ considered in these figures the complete unloading of the cross section after reversed bending occurs under rate elastic behavior, since the maximum residual axial stress is lower than the critical stress $\sigma_{s}$ everywhere. The distributions of the effective Martensite volume fraction $\xi=\xi^{+}-\xi^{-}$along the height plotted in Fig. 4 turn out to be slightly nonlinear within the transforming region, during the first loading process also, although it is very close to a linear trend. Clearly, the total amount of Martensite transformed within the cross section increases with the maximum bending moment $\mu_{\max }$ applied at the end of loading. As the bending moment is completely removed, namely for $\kappa=\kappa_{\text {res }}$, if $\mu<\mu_{\text {lim }}$ then the Martensite distribution does not change (see Figs. $4 a$ and $4 b$ ), being the unloading process entirely elastic, whereas if $\mu>\mu_{\lim }$ then the production of a limited amount of the secondary Martensite variant can be observed (dashed red line in Fig. 4c).

As the bending moment is decreased and then applied with the reversed sign, the size of the region where Martensite reorientation takes place, namely where $\sigma<-\sigma_{s}$, increases. At first it reaches the top surface of the cross section for $\kappa=\kappa_{3}$ and then it tends to recover the entire region where the martensitic transformation occurred under direct bending, namely the upper region over the height $y_{s}$. Note that when the beam curvature is completely removed, namely for $\mathrm{\kappa}=0$ (dashed green lines in Fig. 4), the effective Martensite volume fraction is still positive within the cross section. No backward Austenitic transformation is activated under reversed bending, being $T<A_{s}$. However, a significant amount of the primary (positive) Martensite variant undergoes the reorientation process into the secondary (negative) Martensite variant and thus the effective volume fraction of Martensite $\xi$ decreases. Obviously, a similar but opposite Martensite reorientation occurs in the lower half of the cross section. Also the distributions of the effective Martensite volume fraction $\xi$ at the end of reversed bending for $\kappa=-\kappa_{\max }$ (dash-dotted blue lines) are opposite, but smaller than those observed at the end of direct bending for $\kappa=\kappa_{\max }$ (solid black lines), thus denoting that the initial Martensite variant is still present within the cross section at the end of reversed bending. 
The partition of the cross section at the end of first unloading, namely for $\kappa=\kappa_{\text {res }}$, varying the maximum applied bending moment $\mu_{\max }$ is plotted in Fig. 5. In this plot, the region below the curve $y_{s}$ (solid black line) is in a purely austenitic state; the transformation region between the curves $y_{s}, y_{f}$ (solid blue line), $y^{\prime}$, and $y^{\prime \prime}$ (dashed and das-dotted red lines) is made of a mixture of austenite and single variant Martensite; finally, in the region between the curves $y^{\prime}$ and $y^{\prime \prime}$ the reorientation of the Martensite variant occurred during unloading.

The partition of the cross section at the end of reversed bending, namely for $\kappa=-\kappa_{\max }$, varying the maximum applied bending moment $\mu_{\max }$ is also reported the same plot. In this case, $y^{\prime}=y_{s}$ and $y_{f}<$ $y_{-f, \min }<a<y^{\prime \prime}$. Therefore, the region below the curve $y_{s}$ still is in a purely austenitic state; the region between the curves $y_{s}$ and $y_{-f, \min }$ (dashed green line), is made of a mixture of austenite and both Martensite variants; finally, the region over the curve $y_{-f, \min }$ is entirely made of the secondary Martensite variant. The latter distribution does not change during the subsequent elastic unloading. The closed form relations between the normalized applied bending moment $\mu$ and beam curvature $\kappa$ under the direct and reversed bending cyclic process are plotted in Fig. 6 for $\mu_{\max }=2,3$, and 4 .

Due to the lower Young's modulus of Martensite with respect to that of Austenite, the slope of the last part of the loading process as well as that of the elastic unloading process is clearly smaller than the slope of the initial austenitic loading process. This occurrence makes the residual beam curvature after complete unloading as well as the area enclosed by each cycle a little bit smaller than those obtained under the simplifying assumption of equal Young's moduli of the two phases (Ostadrahimi et al., 2015). Therefore, such a simplifying assumption overestimates the shape memory effect and the energy dissipation capabilities of SMA, as already observed under torsional loading (Radi, 2020).

\subsection{Validation by comparison with available results}

In order to validate the present analysis, a comparison with some analytical results available in the technical literature is provided in Fig. 7. To this aim, the axial stress distributions in a rectangular cross section of heigth $2 a=2 \mathrm{~mm}$ and width $b=2 \mathrm{~mm}$ subject to an increasing bending moment at constant temperature $T=30^{\circ} \mathrm{C}$ given in Fig. $12 a$ of Eshghinejad and Elahinia (2015) (blue lines) are compared with those obtained in the present work (red lines). The constitutive parameters considered by Eshghinejad and Elahinia (2015) at temperature $T=30^{\circ} \mathrm{C}>A_{f}$ are given in Tab. 2. The curves are very close, although a slight discrepancy can be noticed for the higher levels of loading due to the difference between the adopted SMA constitutive models. Eshghinejad and Elahinia (2015) assumed indeed linear stress distributions in all regions, whereas slightly nonlinear stress distributions are 
found in the present analysis, as due to the different Young's moduli of the two phases.

In addition, the tip displacement of a SMA cantilever beam caused by a tip force $F$ in a loadingunloding cycle from 0 to $10 \mathrm{~N}$ obtained from the present formulation is compared in Fig. 8 with that provided by Eshghinejad and Elahinia (2015) in their Fig. 8c. The constitutive parameters at temperature $T=-5^{\circ} \mathrm{C}<A_{s}$ considered therein are reported in Tab. 2 (note that the value assumed for $\varepsilon_{L}$ can not be found in their paper and it has been supplied by the authors by private communication). The tip displacement calculated numerically by double integrating the differential equation for a cantilever Euler-Bernoulli beam, built-in at the end at $x=L=30 \mathrm{~mm}$, namely

$$
w=\frac{L^{2} \sigma_{s}}{a E_{A}} \int_{0}^{1} d \xi \int_{\xi}^{1} \kappa\left(\frac{3 F L \eta}{2 b a^{2} \sigma_{s}}\right) d \eta,
$$

where $\kappa=\kappa(\mu)$ denotes the inverse relation between normalized bending moment and curvature defined in Section 3 for first loading and elastic unloading. In this case also, the results are reasonably close and a discrepancy can be noticed for the higher levels of loading due to the differences between the two SMA constitutive models. In particular, during the martensitic transformation the present model turns out to be a bit stiffer than that developed by Eshghinejad and Elahinia (2015).

A further comparison is made in Fig. 9 with the analytical and FEM results provided by Ostradrahimi et al. (2015) for the bending moment-curvature relation of a rectangular cross section with $a=0.5$ $\mathrm{mm}$ and $b=1 \mathrm{~mm}$. These results are obtained under direct loading and elastic unloading at temperature $T=-40^{\circ} \mathrm{C}$ lower than $M_{s}=-34^{\circ} \mathrm{C}$ and thus also lower than $A_{s}$. The corresponding material parameters are reported in Tab. 3. The analytical model developed by these authors assumes the same Young's moduli for both phases, namely $\Gamma=0$. In this case the stress-strain relations become linear also in the transformation region. The analytical results obtained from the present approach for the particular case of $\Gamma=0$ are practically coincident with those obtained by Ostradrahimi et al. (2015) and very close to the FEM predictions. A slight difference can be observed at the end of elastic unloading because the model considered in Ostradrahimi et al. (2015) allows for reverse austenitic transformation before complete unloading, whereas for the present model the reverse phase transformation can not take place at temperature lower than $A_{s}$.

Unfortunately, no analytical or numerical investigations are available in the technical literature for validating the present model under the reverse bending process, in particular for the temperature range originating the shape memory effect considered here.

\section{Conclusions}

A simple 1D constitutive model is adopted for modelling the isothermal response of a SMA beam 
with rectangular cross section under direct and reversed pure bending. The model takes into account for different Young's moduli of the two phases by adopting the Reuss scheme of composite materials for the description of the SMA elastic behavior. Two opposite Martensite variants can take place according to the sign of the axial stress. Their volumetric fractions are assumed to evolve linearly with the axial stress, so that the linear rate constitutive equations for SMA can be easily integrated in closed form within each step. Moreover, the axial stress and the Martensite distribution across the beam cross section can be calculated analytically at each step of the cyclic process. In particular, the distributions of axial stress and effective Martensite volume fraction in the transformed regions of the cross section are slightly nonlinear as due to the different Young's moduli of the two phases. The analytical or numerical integration of the contribution of the axial stress within the cross section then yields the bending moment applied to the cross section as a function of the beam curvature both under bending and reversed bending.

We found here that the response of the cross section under reversed bending is not symmetrical with respect to the first bending loading, namely the stress field and the Martensite distribution at the end of negative reloading is not exactly opposite to those observed at the end of loading, although a symmetric tension-compression constitutive behavior has been assumed. The difference is due to the different Young's moduli of the two phases. Indeed, the response becomes perfectly symmetric for $\Gamma$ $=0$, namely for $E_{\mathrm{A}}=E_{\mathrm{M}}$. In this case indeed the distributions of stress and Martensite variants at the end of the loading process and at the end of the negative loading process are equal but opposite in sign. With some additional analytical complications, the model can be extended to account also for asymmetric tension-compression behavior of the SMA by assuming different magnitudes of the positive and negative critical stresses.

Here, the analysis has been developed only for the first cycle of direct and reverse loading. In principle, it can be extended to subsequent cycles, of course with increasing difficulties. Future investigations may be extended to multiple loading cycles, general bending, and different temperature ranges. Note that the response during repeated loading cycles of the same amplitude does not change if the elastic modulus of the SMA material is assumed constant, namely for $\Gamma=0$. In this case, indeed, the response observed in the first cycle is exactly reiterated in subsequent cycles of the same amplitude. The present results are also sufficiently accurate under general bending if the shear stress is much smaller than the axial stress, as it generally occurs for slender beam with compact cross section. In this case indeed the shear stress attains the largest values in proximity of the cross section centroid, where no transformation is triggered, and thus a weak effect on the phase transformation condition is expected from the contribution of the shear stress. Finally, the framework used here for modelling Martensite reorientation can be adopted also for modelling the reverse austenitic 
transformation which may occur during unloading at temperatures higher than $A_{s}$.

The exact closed form solution provided here is particularly valuable for analyzing and understanding the role played by the constitutive parameters in the shape memory effect of SMA beams during a bending cycle of reversed sign. Each step of the derivation of the analytical solution is clearly described in the paper and can be easily checked. Besides providing a reliable evaluation of the actual and residual stresses and strains in SMA beam under direct and reverse bending, the analytical results here obtained have a number of additional advantages. Indeed, they can be exploited for the accurate design of innovative seismic dissipating devices and actuators realized by SMA beams loaded in bending and subjected to alternating loading. They can support designers in the calibration process of numerical models able to describe SMA macroscopic effects. They are also useful for validating the accuracy of numerical procedures based on more refined constitutive models employed in the modeling of SMA beams under complex cyclic loading.

\section{Acknowledgements.}

Support from the Italian "Gruppo Nazionale di Fisica Matematica" INdAM-GNFM is gratefully acknowledged.

\section{References}

Auricchio F, Morganti S, Reali A, Urbano M (2011) Theoretical and experimental study of the shape memory effect of beams in bending conditions. Journal of Materials Engineering and Performance 20(4-5): 712-718.

Auricchio F, Sacco E (1997) A Superelastic Shape-Memory-Alloy Beam Model. Journal of Intelligent Material Systems and Structures 8(6): 489-501.

Brinson LC (1993) One-dimensional constitutive behavior of shape memory alloys: thermomechanical derivation with non-constant material functions and redefined Martensite internal variable. Journal of Intelligent Material Systems and Structures 4(2): 229-242.

Brinson LC, Huang MS (1996) Simplifications and comparisons of shape memory alloy constitutive models. Journal of Intelligent Material Systems and Structures 7: 108-114.

Choi E, Lee DH, Choei NY (2009) Shape memory alloy bending bars as seismic restrainers for bridges in seismic areas. International Journal of Steel Structures 9(4): 261-273.

Choi E, Mohammadzadeh B, Kim HS (2019) SMA bending bars as self-centering and damping devices. Smart Materials and Structures 28: 025029. 
De la Flor S, Urbina C, Ferrando F (2011) Asymmetrical Bending Model for NiTi Shape Memory Wires: Numerical Simulations and Experimental Analysis. Strain 47: 255-267

DesRoche R, Smith B (2004) Shape memory alloys in seismic resistant design and retrofit: a critical review of their potential and limitations. Journal of Earthquake Engineering 8: 415-429.

Dolce M, Cardone D, Marnetto R (2000) Implementation and testing of passive control devices based on shape memory alloys. Earthquake Engineering and Structural Dynamics 29: 945-968.

Dolce M, Cardone D (2001) Mechanical behavior of shape memory alloy for seismic applications 1 . Martensite and Austenite NiTi bars subject to torsion. International Journal of Mechanical Sciences 43: 2631-2656.

Eshghinejad A, Elahinia M (2015) Exact Solution for Bending of Shape Memory Alloy Beams. Mechanics of Advanced Materials and Structures 22: 829-838.

Fang C, Wang W (2020) Shape memory alloys for seismic resilience. Springer. Singapore.

Fahimi P, Eskandari AH, Baghani M, Taheri A (2019) A semi-analytical solution for bending response of SMA composite beams considering SMA asymmetric behavior. Composites Part B: Engineering 163: 622-633.

Govindjee S, Kasper ER (1997) A shape memory alloy model for Uranium-Niobium accounting for plasticity. Journal of Intelligent Material Systems and Structures 8: 815-823.

Govindjee S, Kasper ER (1999) Computational aspects of one-dimensional shape memory alloy modeling with phase diagrams. Computational Methods in Applied Mechanics and Engineering 171: 309-326.

Liang C, Rogers CA (1990) One-dimensional thermomechanical constitutive relations for shape memory materials Journal of Intelligent Material Systems and Structures 1(2): 207-234.

Marfia S, Rizzoni R (2013) One-dimensional constitutive SMA model with two Martensite variants: Analytical and numerical solutions. European Journal of Mechanics A/Solids 40:166-185.

Mirzaeifar R, DesRoches R, Yavari A, Gall K (2013) On superelastic bending of shape memory alloy beams. International Journal of Solids and Structures 50: 1664-1680.

Ostadrahimi A, Arghavani J, Poorasadion S (2015) An analytical study on the bending of prismatic SMA beams. Smart Materials and Structures 24: 125035.

Ozbulut OE, Hurlebaus S, Desroches R (2011) Seismic response control using shape memory alloys: a review. Journal of Intelligent Material Systems and Structures 22(14): 1531-1549.

Panoskaltsis VP, Bahuguna S, Soldatos D (2004) On the thermomechanical modelling of shape memory alloys. International Journal of Nonlinear Mechanics 39(5): 709-722. 
Poorasadion S, Arghavani J, Naghdabadi R, Sohrabpour S (2015). Implementation of microplane model into three-dimensional beam element for shape memory alloys. International Journal of Applied Mechanics 7(6): 1550091.

Radi E (2018) Evolution of multiple Martensite variants in a SMA thick walled cylinder under internal pressure. International Journal of Solids and Structures 155: 15-35.

Radi E (2020) Exact solutions for isothermal cyclic torsional loading of a circular SMA bar exploiting the shape memory effect. International Journal of Solids and Structures Submitted.

Rejzner J, Lexcellent C, Raniecki B (2002) Pseudoelastic behaviour of shape memory alloy beams under pure bending: experiments and modelling. International Journal of Mechanical Sciences 44: 665-686.

Rizzoni R, Marfia S (2015) A thermodynamical formulation for the constitutive modeling of a shape memory alloy with two martensite phases. Meccanica 50(4): 1121-1145.

Rizzoni R, Merlin M, Casari D (2013) Shape recovery behaviour of NiTi strips in bending: experiments and modelling. Continuum Mechanics and Thermodynamics 25: 207-227.

Song G, Ma N, Li HN (2006) Applications of shape memory alloys in civil structures. Engineering Structures 28(9): 1266-1274.

Speicher M, Hodgson DE, DesRoche R, Leon RT (2009) Shape memory alloy tension/ compression device for seismic retrofit of buildings. Journal of Materials Engineering and Performance 18: 746-753.

Viet NV, Zaki W, Umer R (2018) Analytical model for a superelastic Timoshenko shape memory alloy beam subjected to a loading-unloading cycle. Journal of Intelligent Material Systems and Structures 29(20): 3902-3922.

Viet NV, Zaki W, Moumni Z (2019) A model for shape memory alloy beams accounting for tensile compressive asymmetry. Journal of Intelligent Material Systems and Structures 30(18-19): 2697-2715.

Wilde K, Gardoni P, Fukino Y (2000) Base isolation system with shape memory alloy device for elevated highway bridges. Engineering Structures 22: 222-229.

Wilson JC, Wesolowsky MJ (2005) Shape memory alloys for seismic response modification: a stateof-the-art review. Earthquake Spectra 21(2): 569-601.

Zaki W (2012) An efficient implementation for a model of Martensite reorientation in martensitic shape memory alloys under multiaxial nonproportional loading. International Journal of Plasticity 37: 72-94.

Zibasokhan H, Behnamfar F, Azhari M (2019) Experimental study of a new pure bending yielding dissipater. Bulletin of Earthquake Engineering 17(7): 4389-4410. 


\section{Appendix A}

The integral in the definition of $m$ in eqn (3.26) can be calculated as

$$
\begin{array}{r}
\int_{1}^{1+\Gamma}\left[(t+\delta)^{2}-p\right]^{2}\left(1+\frac{\delta}{t}\right) d t=\frac{1}{5}\left[(1+\Gamma)^{5}-1\right]+\frac{5}{4} \delta\left[(1+\Gamma)^{4}-1\right]-\frac{2}{3}\left[(1+\Gamma)^{3}-1\right]\left(p-5 \delta^{2}\right) \\
+\left[(1+\Gamma)^{2}-1\right] \delta\left(5 \delta^{2}-3 p\right)+\Gamma(5 \delta-p)\left(\delta^{2}-p\right)+\delta\left(p-\delta^{2}\right)^{2} \ln (1+\Gamma),
\end{array}
$$

where

$$
p=(1+\delta)^{2}-2 \frac{\Gamma}{\beta}
$$




\section{TABLES}

\begin{tabular}{|c|c|c|c|c|c|}
\hline$T$ & $E_{\mathrm{A}}$ \\
{$\left[{ }^{\circ} \mathrm{C}\right]$} & {$[G P a]$} & $\begin{array}{c}E_{\mathrm{M}} \\
{[G P a]}\end{array}$ & $\begin{array}{c}\sigma_{s} \\
{[M P a]}\end{array}$ & $\begin{array}{c}\sigma_{f} \\
{[M P a]}\end{array}$ & $\varepsilon_{L}$ \\
\hline 25 & 67 & 26.3 & 153 & 223 & 0.067 \\
\hline
\end{tabular}

Table 1. Young's moduli of the two phases, Poisson coefficient, critical stresses and maximum residual transformation strain for Brinson SMA materials at room temperature.

\begin{tabular}{|c|c|c|c|c|c|}
\hline $\begin{array}{c}T \\
{\left[{ }^{\circ} \mathrm{C}\right]}\end{array}$ & $\begin{array}{c}E_{\mathrm{A}} \\
{[G P a]}\end{array}$ & $\begin{array}{c}E_{\mathrm{M}} \\
{[G P a]}\end{array}$ & $\begin{array}{c}\sigma_{s} \\
{[M P a]}\end{array}$ & $\begin{array}{c}\sigma_{f} \\
{[M P a]}\end{array}$ & $\varepsilon_{L}$ \\
\hline-5 & 73.2 & 30 & 25 & 125 & 0.0166 \\
\hline 30 & 73.2 & 30 & 200 & 300 & 0.0166 \\
\hline
\end{tabular}

Table 2. SMA constitutive parameters at temperatures $T=-5^{\circ} \mathrm{C}$ and $T=30^{\circ} \mathrm{C}$, from Eshghinejad and Elahinia (2015).

\begin{tabular}{|c|c|c|c|c|c|}
\hline $\begin{array}{c}T \\
{\left[{ }^{\circ} \mathrm{C}\right]}\end{array}$ & $\begin{array}{c}E_{\mathrm{A}} \\
{[G P a]}\end{array}$ & $\begin{array}{c}E_{\mathrm{M}} \\
{[G P a]}\end{array}$ & $\begin{array}{c}\sigma_{s} \\
{[M P a]}\end{array}$ & $\begin{array}{c}\sigma_{f} \\
{[M P a]}\end{array}$ & $\varepsilon_{L}$ \\
\hline-40 & 53 & 53 & 122.5 & 197.5 & 0.05 \\
\hline
\end{tabular}

Table 3. SMA constitutive parameters at temperatures $T=-40{ }^{\circ} \mathrm{C}$ from Ostradrahimi et al. (2015). 


\section{FIGURES}

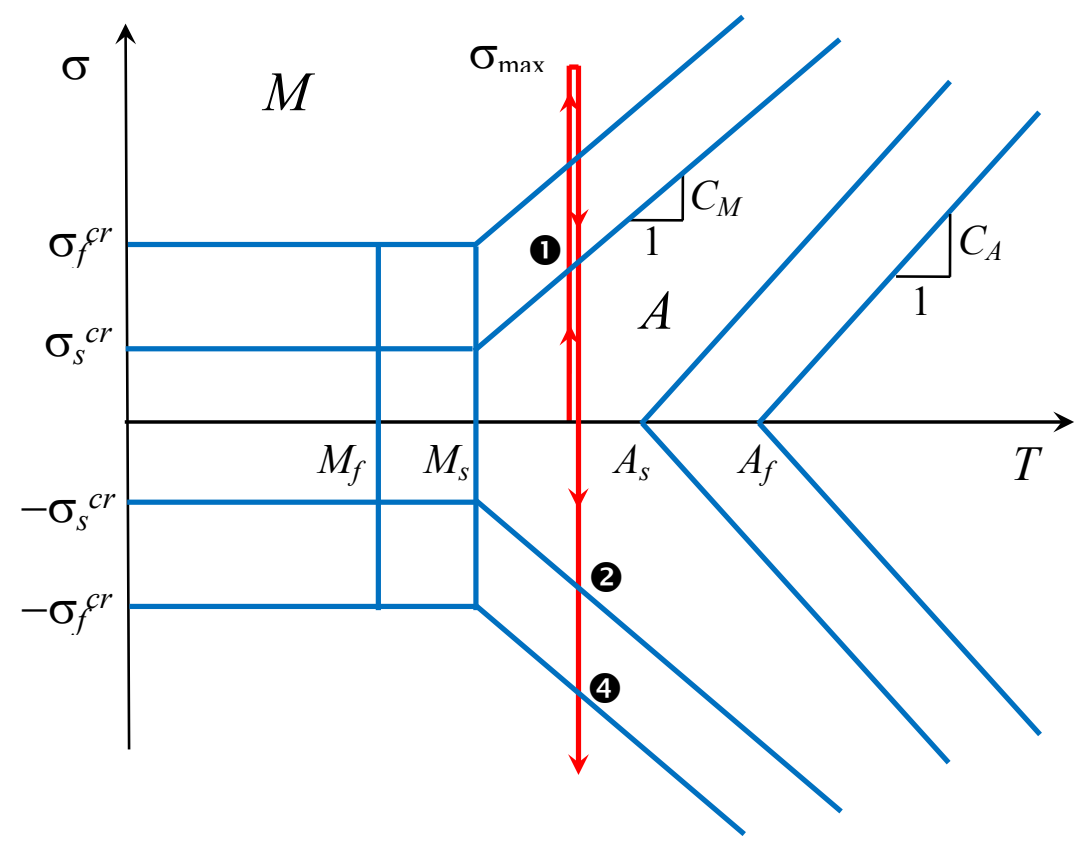

Fig 1. Threshold stresses for martensitic and austenitic transformations (blue lines) and isothermal loading-unloading process (red lines) at temperature $T$ between $M_{S}$ and $A_{s}$.

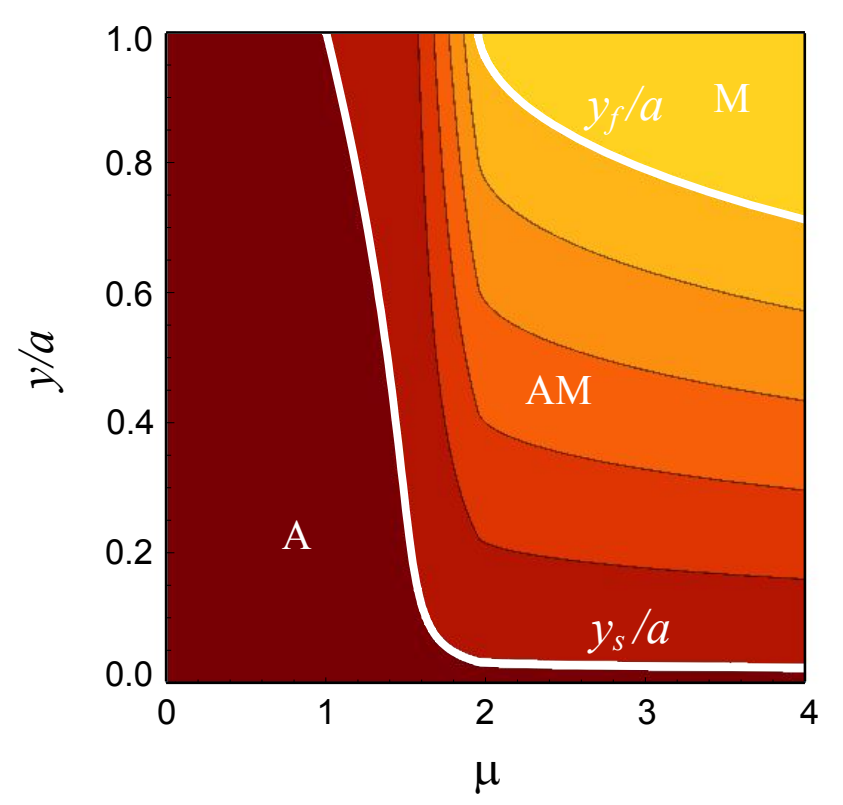

Fig. 2. Distribution of Austenite and Martensite and variations of the fronts of finish and start martensitic transformation with the applied bending moment under loading, for Brinson SMA material. 

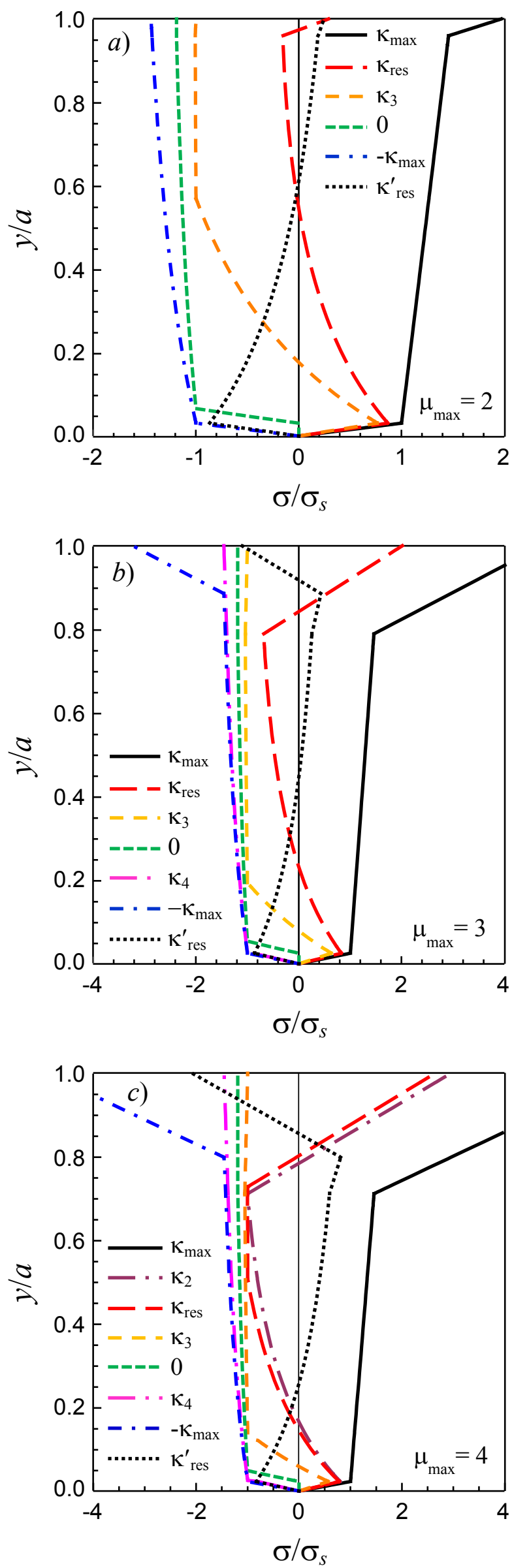
Fig. 3. Distributions of the axial stress $\sigma$ along the height of the cross section, for various values of the beam curvature $\kappa$ and for $\mu_{\max }=3,4$, and 5, for Brinson SMA material. 

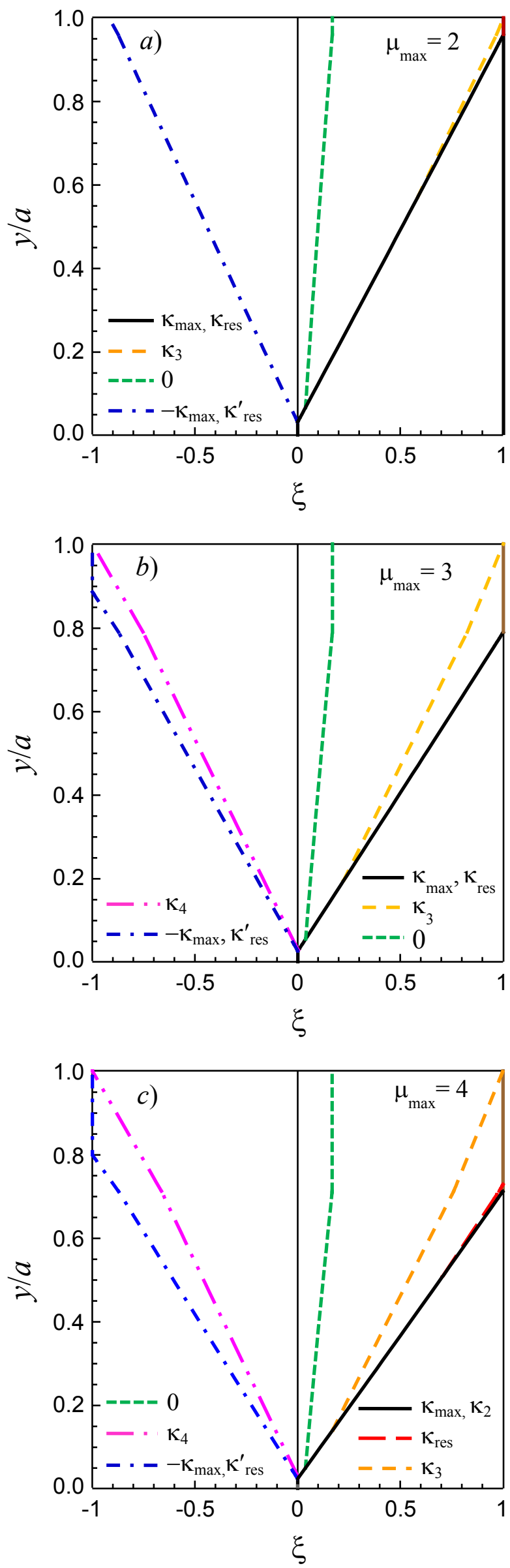
Fig. 4. Distributions of the effective Martensite volume fraction $\xi$ along the height of the cross section, for various values of the beam curvature $\kappa$ and for $\mu_{\max }=3,4$, and 5, for Brinson SMA 


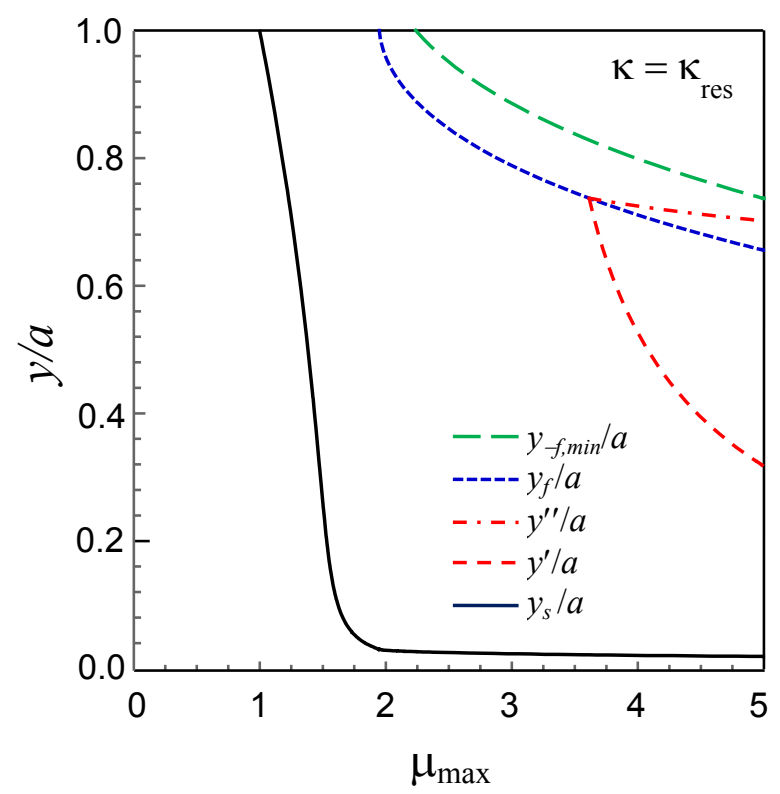

Fig. 5. Normalized variations with the maximum bending moment applied to the cross section of the fronts of finish and start martensitic transformation and of the upper and lower fronts of the region undergoing transformation between Martensite variants at first complete unloading and at the end of reversed bending, for Brinson SMA material.

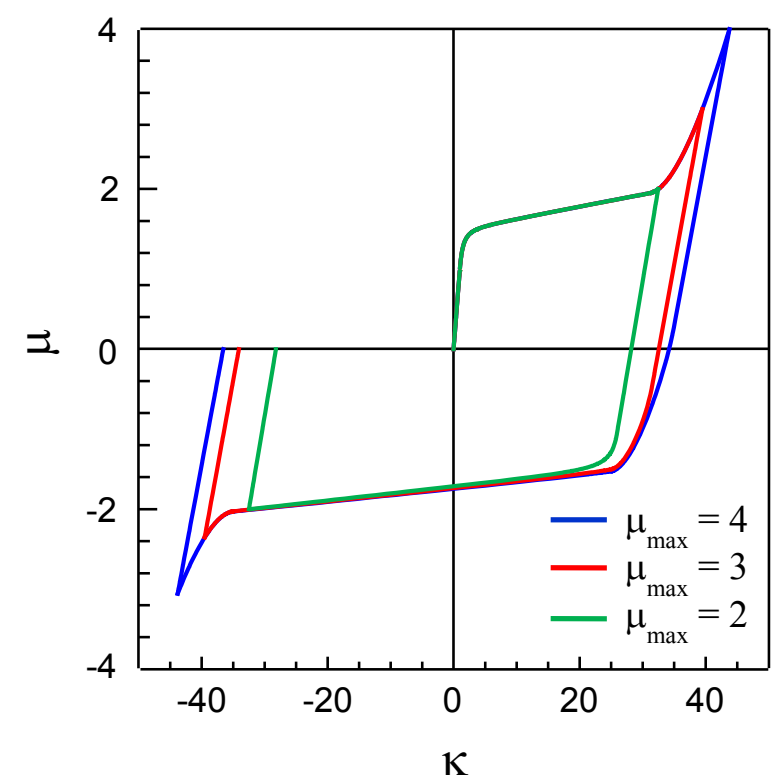

Fig. 6. Normalized cyclic variations of the applied bending moment with the beam curvature, under alternative loadings for $\mu_{\max }=2,3$, and 4, for Brinson SMA material. 


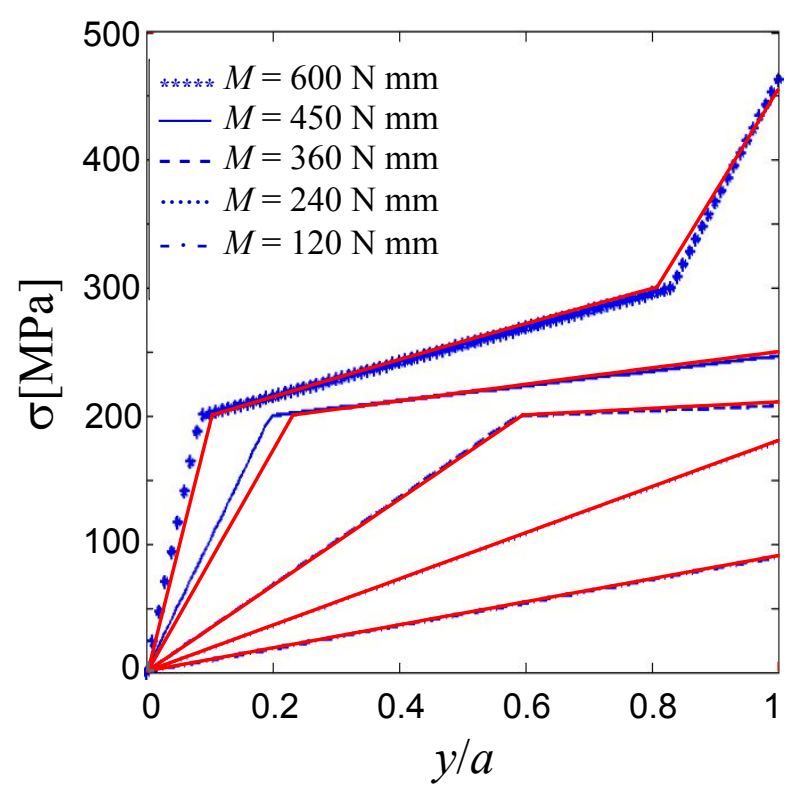

Fig. 7. Comparison between the axial stress distributions along the height of the beam provided by the present analysis (red lines) and those provided in Fig. 12a of Eshghinejad and Elahinia (2015) (blue lines) for Material I, for the loading process at temperature $T=30^{\circ} \mathrm{C}$.

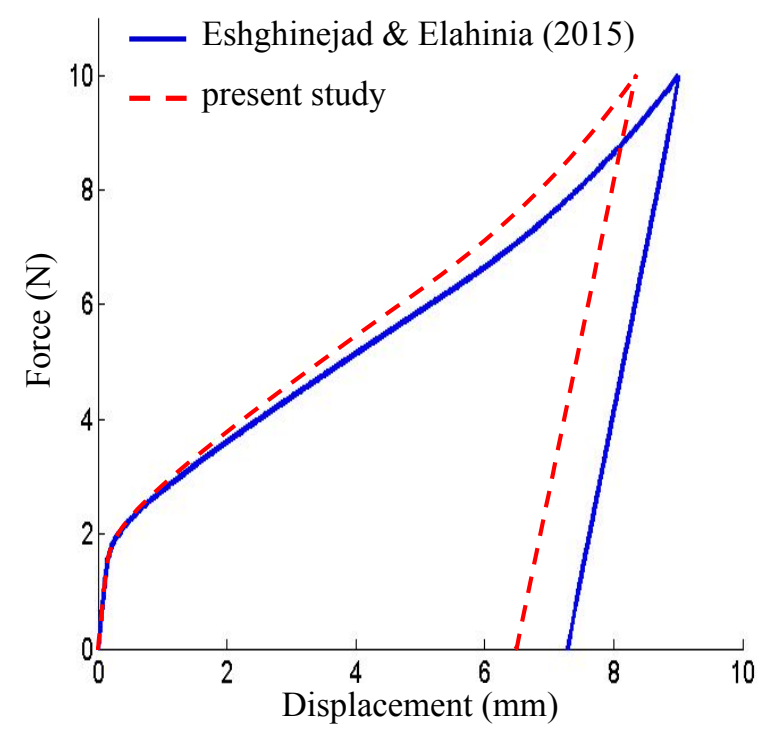

Fig. 8. Comparison between the force vs tip displacement relation of a SMA cantilever beam at $T=$ $-5^{\circ} \mathrm{C}$ provided by the present analysis (dashed red line) and those provided in Fig. $8 c$ of Eshghinejad and Elahinia (2015) (solid blue line). 


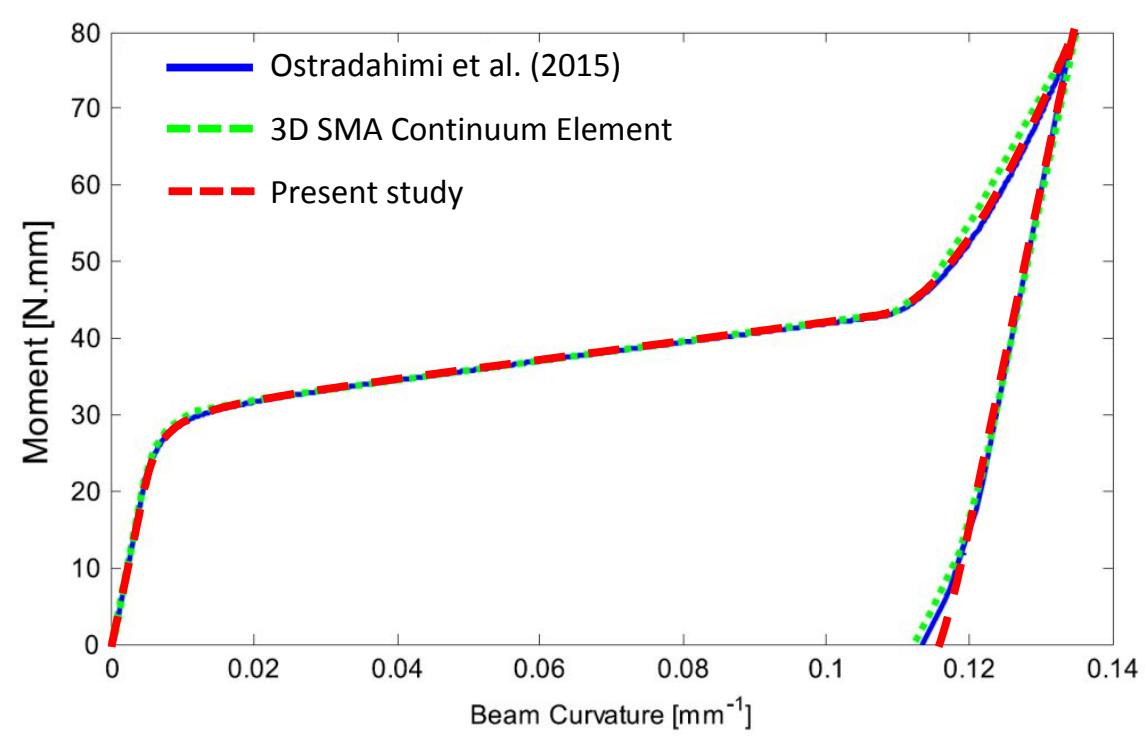

Fig. 9. Comparison between the moment-curvature relation of a SMA rectangular cross section with $a=0.5 \mathrm{~mm}$ and $b=1 \mathrm{~mm}$ provided by the present analysis (dashed red line) and those provided in Fig. 12 of Ostradrahimi et al. (2015), obtained by their analytic model (solid blue line) and by FEM analysis (dashed green line). 